\title{
Lot sizing problem with batch ordering under periodic buyback contract and lost sales
}

\author{
Mlouka Farhat ${ }^{\mathrm{a}, \mathrm{c}}$, Ayse Akbalik ${ }^{\mathrm{b}}$, Atidel B. Hadj-Alouane ${ }^{\mathrm{c}}$, Nathalie Sauer ${ }^{\mathrm{a}}$
}
${ }^{a}$ Université de Lorraine, LGIPM, Technopôle, 57073 METZ, France (e-mail: \{mlouka.farhat, nathalie.sauer\}@ univ-lorraine.fr)
${ }^{b}$ Université de Lorraine, LCOMS, Technopôle, 57073 METZ, France (e-mail: ayse.akbalik@univ-lorraine.fr)
${ }^{c}$ Université de Tunis El Manar, OASIS, École Nationale d'Ingénieurs de Tunis, BP 37 Le Belvédère, 1002 Tunis, Tunisie
(e-mail: atidel.hadj@enit.utm.tn)




\title{
Lot sizing problem with batch ordering under periodic buyback contract and lost sales
}

\begin{abstract}
We study the deterministic single-item lot sizing problem with batch ordering under the buyback contract (LSP-BB) in a system with one retailer and one supplier. We consider a fixed cost per batch replenished in addition to the classical lot sizing costs, making the procurement cost structure stepwise. We also consider lost sales option with a lost sales cost incurred for each unit of demand not satisfied. The buyback contract considered here consists in returning unused units at the end of every $w$ periods, with a buyback revenue for each unit returned back. We study this problem under both FTL (full truck load) cost structure and only full batch replenishment assumption. We propose efficient and exact polynomial time algorithms for different extensions of this problem which is known to be NP-hard in the general case. To the best of our knowledge, it is the first time that the buyback contract is introduced in a multi-period lot sizing problem (LSP) in the literature. Moreover, batch ordering and lost sales issues have never been tackled in the same LSP model before our study. Hence, this paper provides efficient algorithms to solve the LSP with batch ordering and lost sales under the buyback contract not yet addressed in the literature.
\end{abstract}

Keywords: Lot sizing, buyback contract, batch delivery, piecewise cost, polynomial algorithm, lost sales. 


\section{Introduction}

We are interested in a single-item lot sizing problem (LSP) where a retailer signs a buyback contract with a supplier who provides him the quantities in batches (e.g. pallets, containers or trucks). The classical LSP consists in planning production (or replenishment) and storage activities at minimum cost in order to satisfy deterministic and discrete demand over a finite horizon. The main costs are setup (or fixed ordering cost), unit production (or procurement) cost and holding cost. LSP has several extensions in the literature : with a limited capacity, multi-item, multi-level, with lost sales, or backlogging, etc. (see Brahimi et al. 2017). Several exact and heuristic methods have been proposed to solve them. We study this problem integrating buyback contract, with and without lost sales option while considering two different cost patterns: FTL (with a possibility of fractional batch replenishment) or OFB (only full batch) replenishment. The buyback contract is a commitment in which the buyer has the possibility to return a certain percentage of the unused items at the end of the selling season to the supplier for a revenue less than or equal to the procurement cost. In the literature, the buyback contract is mostly used in single period planning problems occurring in the supply chains. According to Hou et al., (2010), there are three factors that lead to use this type of contract: demand pattern (uncertainty, price dependence, etc.), properties of the product (perishability, single or multi-item patterns, etc.), and supply chain structure. In our problem, we consider a multi-period setting and we suppose that the retailer has to return

all the unused products (called also full return) to the supplier at the end of 
every $w$ periods. For instance, a period can represent a day, and returns can be operated only at the end of each week $(w=7)$.

The buyback contract arises in many industrial contexts: any product with a limited life due to physical decay (dairy products, baked goods, pharmaceuticals, cosmetics) and products with a risk of obsolescence (fashion apparel, computer hardware and software, greeting cards, magazines, newspapers). The shorter the product lifetime and the higher the inventory costs, the more the LSP-BB with a known $w$ becomes interesting to solve. For instance, McKesson, a major distributor of health and beauty products, offers retailers a return program trading off more generous return policies at higher purchase prices (Padmanabhan and Png, 1995). The determination of the parameter $w$ depends on the life of the product and the cost of carrying inventory, so it can be variant on the planning horizon. Another example is Wahmpreneur Books which sells books to retailers and wholesalers which may return them for full credit within a 30 days timeline (Bose and Anand, 2007).

This paper also presents two important variants of the batch replenishment: the first one is that the ordered quantity in each period $t$ is restricted to a multiple of a certain batch size $V_{t}$ (called only full batch - OFB) and the other is that the replenished batch can be incomplete (stepwise cost structure, FTL cost). We consider a general form of procurement cost structure that includes a fixed cost for each order (classical setup cost), a variable unit purchase cost, and a fixed cost per batch replenished that reflects stepwise cost function composed by flat sections of size $V_{t}$ (see Figures 2 and 3 ).

Furthermore, our study adds an important dimension where the retailer 
allows for lost sales on some periods over the horizon. Demand of such periods can be entirely or partially lost. According to Bijvank and Vis (2011), in case of stock-out of a specific product, the majority of the customers will not wait and will rather buy a different product or visit another store. Thus, in practical settings, the original demand can be considered to be lost, even if it is quite common to consider the backlogging of the excess demand in the classical inventory literature. Contrary to the lost sales, backlogging aims to satisfy the demand, but later than the initially required period. Usually, the backlog also incurs a penalty cost, either proportional to the amount backlogged, or to the duration of backlog (see Pochet and Wolsey, 2006). Hence, the present paper establishes efficient algorithms to solve an LSP with both batch ordering and lost sales, under the buyback contract, not yet addressed in the literature. In the rest of the paper, the reader will discover that our buyback assumption does not make the problem difficult, on the contrary, it will allow us to decompose the problem into smaller subproblems. However, the lost sales assumption can increase the overall complexity of the problem by increasing the number of feasible production plans. See in Table 1 different cases studied in this paper with the respective computational complexities.

The rest of this paper is organized as follows. Section 2 presents some relevant studies related to LSP with and without batch ordering, buyback contracts and LSP with lost sales, stating the closest ones to our studies. Section 3 is dedicated to the description of the LSP-BB studied in this paper, and proposes a mathematical formulation by a mixed integer linear program. Section 4 is dedicated to LSP-BB without lost sales, under OFB or FTL 
Table 1: Complexities of all the algorithms for different cases studied in this paper.

OFB: only full batch, FTL: full truck load, $T$ : length of the planning horizon, $w$ : periodicity of the return periods

\begin{tabular}{|l||l|l|l|l|l|l|l|l|}
\hline \multirow{2}{*}{ Hypotheses } & \multicolumn{3}{c|}{ Without lost sales } & \multicolumn{4}{c|}{ With lost sales } \\
\cline { 2 - 9 } & \multicolumn{2}{|c|}{ OFB } & \multicolumn{2}{c|}{ FTL } & \multicolumn{2}{c|}{ OFB } & \multicolumn{2}{c|}{ FTL } \\
\cline { 2 - 9 } & $w=1$ & $w>1$ & $w=1$ & $w>1$ & $w=1$ & $w>1$ & $w=1$ & $w>1$ \\
& with & with & with & with & with & with & with & with \\
& $V_{t}$ & $V$ & $V_{t}$ & $V$ & $V_{t}$ & $V$ & $V_{t}$ & $V$ \\
\hline Complexity & $O(T)$ & $O(T w)$ & $O(T)$ & $O\left(T w^{3}\right)$ & $O(T)$ & $O\left(T w^{3}\right)$ & $O(T)$ & $O\left(T w^{3}\right)$ \\
\hline
\end{tabular}

patterns. Exact polynomial time algorithms are proposed for the case with $w=1$, and followed by the general case with $w>1$. Section 5 deals with the LSP-BB under lost sales, and presents respective dynamic programming (DP) algorithms for different extensions. Section 6 concludes the paper and discusses some possible perspectives of this work.

\section{Literature review}

In this section, we position our research within the lot sizing problem and procurement contracts literature. We categorize the relevant literature into four groups according to their subject areas: classical LSP, LSP with batch ordering (including piecewise or stepwise cost functions), buyback contracts (also more general ones), and LSP with lost sales. We begin by comparing our work to the closest studies in the literature, to better state our contributions before detailing the related literature.

\subsection{Closest papers to our study and our contributions}

Our contributions in the literature are shown in Figure 1 with vertical lines and they will be detailed below. 


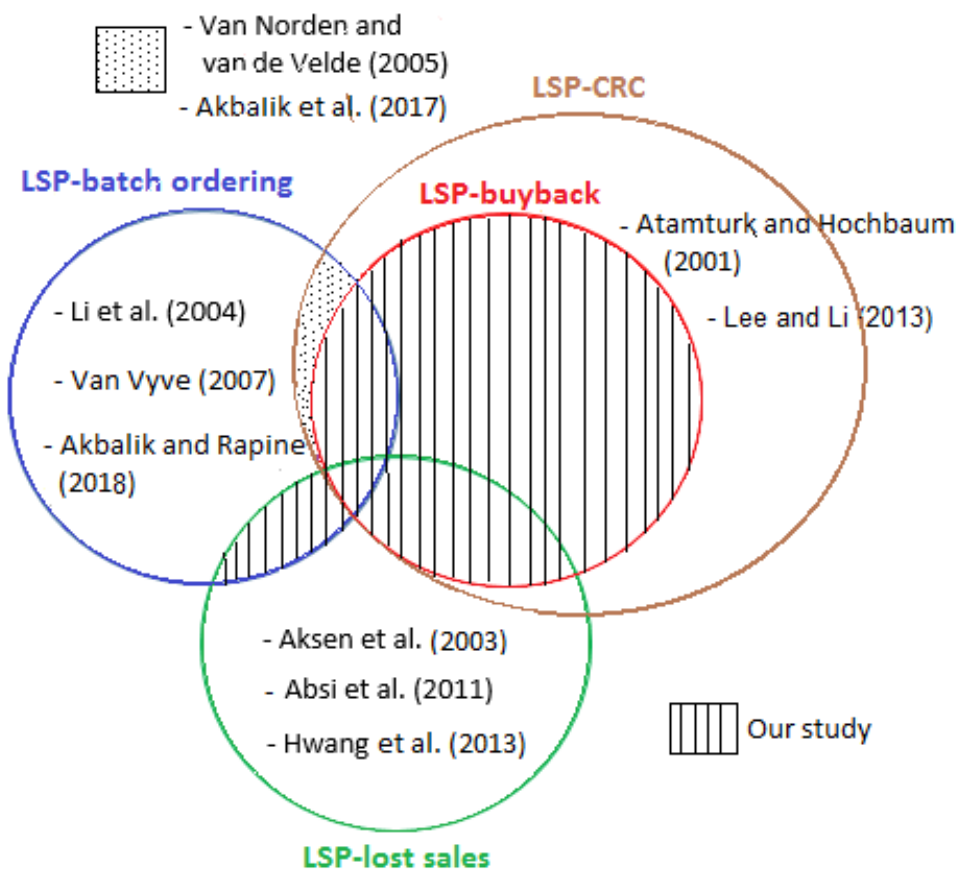

Figure 1: The contributions of this study

The first contribution of this article is to solve the LSP under the buyback contract, which is, to the best of our knowledge, not yet explored in the literature. However, there are a few papers on the LSP under the capacity reservation contract $(\mathrm{CRC})$, which consists in a general form of the buyback contract (Atamturk and Hochbaum (2001), van Norden and van de Velde (2005), Lee and Li (2013), Akbalik et al. (2017)).

In addition, the total procurement cost structure considered in this paper is stepwise, being studied by many papers in the literature (e.g. Li et al. (2004), Akbalik and Rapine (2018)) but without buyback contract nor lost sales. To the best of our knowledge, the batch ordering problem has never been addressed in the literature integrated with lost sales. 
Another contribution of this paper is on the LSP with lost sales literature. There are a few papers making lost sales assumption for LSP (Aksen et al. (2003), Absi et al. (2011), Hwang et al. (2013)) but without batch ordering. Thus, the third particularity of our problem is to solve this LSP both with batch ordering and lost sales conditions.

\subsection{Classical LSP}

The aim of the LSP is to decide in which periods and which quantities to produce (or to procure in our case) over a finite horizon of $T$ periods in order to satisfy the demand while minimizing the production and the inventory holding costs. The first work on discrete and deterministic uncapacitated LSP (ULSP) was developed by Wagner and Whitin (1958) proposing an algorithm in $\mathrm{O}\left(T^{2}\right)$ time which has been improved by Wagelmans et al. (1992), Federgruen and Tzur (1991), and Aggarwal and Park (1993), to an $O(T \log (T))$ time algorithm in the general case and to an $O(T)$ time algorithm under the non-speculative costs. The papers of Florian et al. (1980), and Bitran and Yanasse (1982) prove the complexity of the single-item capacitated LSP (CLSP) depending on the structure of setup, inventory and production costs and capacities. For more details on the LSP, refer to Karimi et al. (2003), Buschkühl et al. (2010) and Brahimi et al. (2017).

\subsection{LSP with batch ordering}

As mentioned by Koca et al. (2014), piecewise or stepwise procurement cost functions can be used to represent quantity discounts, capacity acquisition, subcontracting, overloading, minimum quantity requirements and capacities, as well as a combination of these latters. In our study, we consider a 
stepwise cost function, also called FTL (Akbalik and Pochet (2009), Akbalik and Rapine $(2012,2013))$ under which products are ordered at period $t$ in batches and the customer pays the cost $a_{t}$ for a full batch of $V_{t}$ units even if the batch is not complete. The total procurement cost is composed of a fixed setup cost $f_{t}$, a unit purchase cost $p_{t}$, and of a fixed cost $a_{t}$ per batch replenished. Figure 2 illustrates this stepwise cost structure for null unit cost and Figure 3 for the additional unit procurement cost. For a complete literature survey, the readers are referred to Akbalik and Rapine (2013). In the same paper, the authors show that for time-dependent batch sizes, the problem is NP-hard when one of the cost parameters is also time-dependent. A special case of our problem is thus NP-hard.

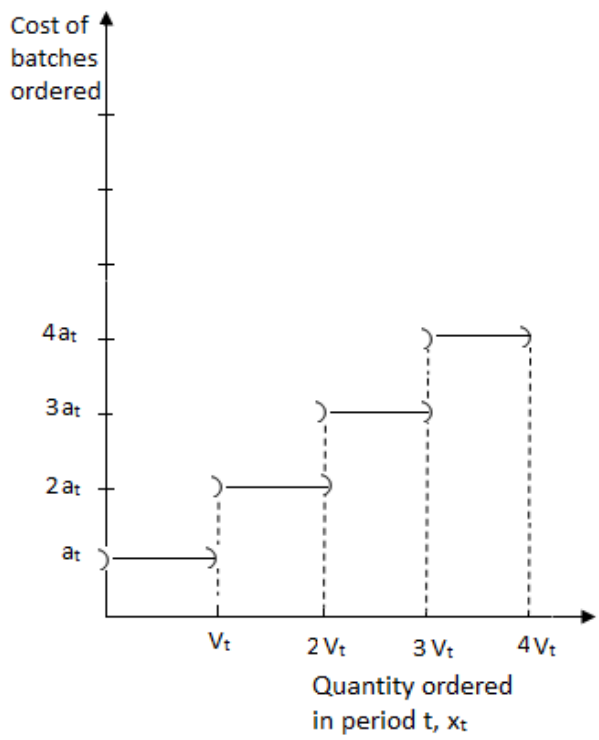

Figure 2: Full-truckload cost function

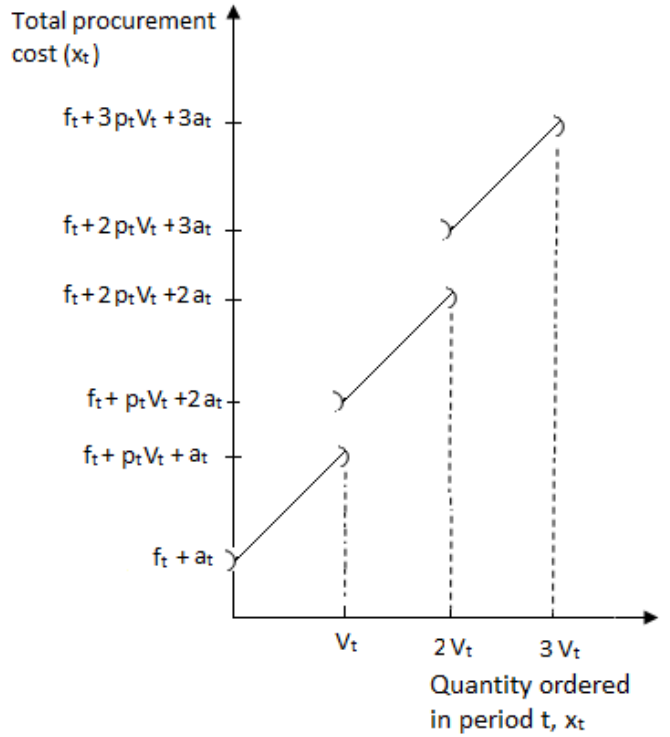

Figure 3: Total procurement cost function (assumed in this study)

Li et al. (2004) study ULSP with batch ordering with setup cost, time varying cost parameters and backlogging. In the first problem, they develop 
an algorithm that solves the dynamic LSP with multiple of a constant batch size ordering in $\mathrm{O}\left(T^{2}\right)$ time improved to $O(T \log (T))$ time through the use of Monge matrices. An $\mathrm{O}\left(T^{3} \log (T)\right)$ time algorithm is also developed by the same authors to solve the LSP with Truck Load discounts. Akbalik and Rapine (2018) study the ULSP with multi-mode replenishment and batch deliveries. They allow incomplete batches but the same fixed cost is paid whatever the number of items in a batch. Besides, they show that this problem is NP-hard. Thereafter, they propose an $O\left(T^{7}\right)$ time algorithm for a special case with stationary batch sizes, only 2 modes and divisible batch sizes, and an $O\left(T^{4}\right)$ time algorithm to solve the single mode problem.

\subsection{Buyback contract}

The best known forms of contracts in the supply chains include longterm contracts, wholesale price contracts, sales rebate contracts, quantity discount contracts, contracts with option and capacity reservation contracts. This paper is concerned with a special type of capacity reservation contract: buyback contract. In a buyback contract the buyer procures $Q$ units for a fee $p$, and receives a revenue, $p^{b}$, for each unit not utilized. This is analogous to a capacity reservation contract with a reservation and procurement of $Q$ units at a cost $p-p^{b}$, and a procurement of $Q-N$ units of excess quantity

for a fee, $p^{b}$ ( $N$ is capacity not utilized).

In several sectors, an industrial company signs a contractual agreement with a supplier that allows for reserving a certain capacity in advance and buying it for an advantageous price. However, if this industry requires an amount that exceeds the reserved capacity to meet the additional demand, it can get it at another price. The capacity reservation contract is often used for 
risk sharing between the supply chain members, in different industries ( $\mathrm{Wu}$ et al. (2005), Hazra and Mahadevan (2009), Park and Kim (2014)). According to Park and Kim (2014), the capacity reservation contracts can be classified into two groups: general contracts which are frequently used in retail and manufacturing industries, and specialized contracts, which are employed in capital intensive industries. The general contracts include quantity flexibility, backup agreement, buyback, minimum commitment, and revenue-sharing contracts. The specialized contracts include take-or-pay, pay-to-delay, and deductible reservation contracts. Concerning the integration of capacity reservation contracts and lot sizing problem, see Atamturk and Hochbaum (2001), van Norden and van de Velde (2005), Lee and Li (2013) and Akbalik et al. (2017).

A buyback (return policies) contract involves three parameters $\left(p, p^{b}, \rho\right)$, with $p$ being the procurement cost per unit, $p^{b}$ being a pay back revenue $(0<$ $\left.p^{b} \leq p\right)$, and $\rho$ being the maximum return percentage $(0<\rho \leq 1)$. Under such a mechanism, the supplier sells $x$ units to a buyer at a cost $p$ per unit and allows the customer to return a maximum of $\rho x$ at the end of the selling season with a revenue $p^{b}$ per unit. The seminal work regarding buyback contracts is performed by Pasternack (1985) for a coordination perspective by maximizing total profits of the retailer and the manufacturer. In a large number of papers (Krihnan et al. (2004), He and Zhao (2012), Wu (2013), etc), the buyback contract has been studied from a design and implementation point of view in different supply chain configurations and not for production planning. In this paper, we will integrate the buyback contract in a multi-period production planning problem. 


\subsection{LSP with lost sales}

Lost sales mean unsatisfied demands where the retailer has to decide to lose either the entire demand or only a portion within a period. It is possible to serve only one part of the demand, if this is more beneficial. Companies having low demand for certain products with low profit in a certain period can find it more profitable to lose this demand. Note that, in lost sales practice, demand cannot be backlogged (Aksen et al., 2003). In lot sizing, the classical decision of how much and when to order is extended to the decision of when and which quantities to lose over the horizon.

A few papers on lot sizing problems with lost sales are presented in the literature. Sandbothe and Thompson (1990) study the ULSP including lost sales. They propose an $\mathrm{O}\left(T^{3}\right)$ time dynamic programming (DP) algorithm to solve it optimally. Aksen et al. (2003) improve the previous complexity to $\mathrm{O}\left(T^{2}\right)$. Hwang et al. (2013) provide algorithms for the ULSP with upper bounds on stocks and lost sales, and the problem with only lost sales considering different cost structures. In Absi et al. (2011), two ULSP with production time windows are studied. The first one allows to integrate lost sales and early production, and the second problem focuses on inclusion of backlogs and early production. They propose two DP algorithms to solve these problems in $\mathrm{O}\left(T^{2}\right)$ time.

\section{Description of the LSP-BB, hypotheses and mathematical for- mulation in MILP}

In the system we consider, a retailer is replenished by batches of a singleitem from an external supplier, where both parts sign a buyback contract. 
At the beginning of each period $t$, the retailer can purchase a certain amount denoted by $x_{t}$ with a known demand $d_{t}$ over a planning horizon of $T$ periods. In real life applications, the batches can correspond to pallets, containers or trucks. Hence, we introduce the variable $A_{t}$ which represents the number of batches ordered in period $t$. A batch can hold up to $V_{t}$ units in period $t$. The retailer accepts to pay a cost of $a_{t}$ for each batch replenished from the supplier in addition to a unit procurement cost $p_{t}$. If there is an order in period $t$, a fixed ordering cost $f_{t}$ is incurred. An inventory holding cost $h_{t}$ is paid for each unit remaining in stock at the end of period $t$. We denote by $s_{t}$ the stock level at the end of period $t$. Without loss of generality, we assume that the initial inventory at the beginning of period 1 is zero. Otherwise, the positive quantity in stock can be retrieved from the demands of the first periods, till obtaining null entering inventory.

In the buyback contract we consider, the return of unused products is made at the end of every $w$ periods with $w \geq 1$. In classical settings of the literature, the quantity to be returned to the supplier is at the end of the selling season in a stochastic single-period problem. In general, under such contracts, the supplier allows the retailer to return a maximum of $\rho x$ units, with $x$ being the quantity purchased at a price $p$ per unit and $\rho$ being the maximum return percentage $(0 \leq \rho \leq 1)$, at a certain return revenue $p^{b}$ with $0 \leq p^{b} \leq p$. If $p^{b}=p$, the contract is said to be full refund, otherwise it is called partial refund. If $\rho=1$, the retailer can return all the unsold units so the contract is said to be full return. Otherwise $(\rho<1)$, the retailer can only return a limited number of unsold units to the supplier, so the contract is said to be partial return. We assume a full return buyback contract with 
partial refund. Our buyback contract involves $q_{t}$ and $p_{t}^{b}$, with $q_{t}$ being the returned quantity at the end of period $t$ which is a decision variable and $p_{t}^{b}$ being the unit return revenue in period $t$ which is a given parameter.

In addition to the quantities and costs mentioned above, we introduce two notations of lost sales $\left(L_{t}, l_{t}\right)$, with $L_{t}$ being the lost sales quantity in period $t$ and $l_{t}$ being the unit lost sales cost in period $t$. We consider the assumption of Aksen et al. (2003) which asserts that the gross marginal profit $\left(l_{t}-p_{t}\right)$ is nonnegative for each $t=1, \ldots, T$, but at the same time we have to take into account the batch ordering cost. Hence, we can make the following assumption without loss of generality: $l_{t} V_{t} \geq p_{t} V_{t}+a_{t}, \forall t=1, \ldots, T$, which means that losing an entire batch incurs a cost greater than or equal to procuring it. If we have $l_{t} V_{t}<p_{t} V_{t}+a_{t}$ in a period $t$, the retailer never orders in that period. Table 2 summarizes the notations that are used.

In this paper, we study two main cases: LSP-BB without lost sales, which means that demands are to be entirely satisfied, and LSP-BB with lost sales. Thereafter, for each of these two problems, we study the following sub-cases:

- Sub-case 1 noted by LSP-BB ${ }_{O F B}$ : The supplier delivers the items only in full batches, which means that the ordered quantity in every period of the planning horizon should be expressed as a multiple of the batch sizes.

- Sub-case 2 noted by LSP-BB ${ }_{F T L}$ : Without any restriction on the batch status, which means an FTL cost structure. In a period $t$, the batches replenished can be fractional.

For each case and sub-case, we consider two return policies. The first one 
Table 2: Notations for the general model of the LSP-BB

\begin{tabular}{|c|c|}
\hline \multicolumn{2}{|c|}{ Parameters } \\
\hline$T$ & length of the horizon \\
\hline$w$ & periodicity of the return periods \\
\hline$p_{t}$ & unit procurement cost (variable cost) in period $t$ \\
\hline$f_{t}$ & fixed ordering cost (setup cost) in period $t$ \\
\hline$a_{t}$ & fixed cost per batch replenished in period $t$ \\
\hline$h_{t}$ & inventory holding cost per unit at the end of period $t$ \\
\hline$p_{t}^{b}$ & unit return revenue in period $t$ \\
\hline$l_{t}$ & unit lost sales cost in period $t$ \\
\hline$d_{t}$ & demand in period $t$ \\
\hline$V_{t}$ & batch size in period $t$ \\
\hline \multicolumn{2}{|c|}{ Decision variables } \\
\hline$x_{t}$ & amount of procurement in period $t$ \\
\hline & $\int 1$ if a procurement takes place in period $t$ \\
\hline $\mathrm{y}_{t}$ & $\left\{\begin{array}{l}\text { otherwise } \\
0\end{array}\right.$ \\
\hline$A_{t}$ & number of batches ordered in period $t$ \\
\hline$s_{t}$ & $\begin{array}{l}\text { stock level (quantity remaining in stock) at the end of } \\
\text { period } t\end{array}$ \\
\hline$q_{t}$ & $\begin{array}{l}\text { returned quantity of unused products at the end of pe- } \\
\text { riod } t\end{array}$ \\
\hline$L_{t}$ & $\begin{array}{l}\text { lost sales quantity (amount of unmet demand) in period } \\
t\end{array}$ \\
\hline
\end{tabular}

is the LSP-BB with $w=1$ which means that the product return is made at the end of each period $t$. For instance, in the Hungarian market, the retailers return the unsold books $(\rho=1)$ at the end of each month without any or with a small charge (Dobos and Wimmer, 2010). The second one is the LSP-BB with $w>1$, which means that the return is at the end of every $w$ periods with $w>1$. For example, at the end of summer, Procter \& Gamble, an American manufacturer of hygiene and beauty products, refunds its retailers the unsold inventory value of Bain-de-soleil $(\rho=1)$ which is a sun care product line (Padmanabhan and Png, 1997). In total, we study 8 problems, see Table 1. 


\section{Assumptions}

The LSP-BB without and with lost sales have one general assumption as follows:

- $\quad \rho=1$ (full return), see examples in page 14 .

In addition, the LSP-BB with lost sales has the following assumption, without loss of generality:

- $\quad l_{t} V_{t} \geq p_{t} V_{t}+a_{t}, \forall \mathrm{t}=1, \ldots, T$, see page 13 .

Moreover, for the LSP-BB with $w=1$, we consider the following assumptions:

- Batches with time-dependent sizes $\left(V_{t}\right)$.

- $p_{t}^{b}<p_{t}, \forall \mathrm{t}=1, \ldots, T$, this is a criterion of the buyback contract, see page 10 .

In contrast, for LSP-BB with $w>1$, we assume:

- $\quad$ Constant batch size $(V)$, see page 8 .

- $\quad p_{i w}^{b}<p_{t}, \forall t \in\{(i-1) w+1, \ldots, i w\}$ and $\forall i \in\left\{1, \ldots, \frac{T}{w}\right\}$ with $i \in \mathbb{N}^{*}$.

- Non-increasing (NI) lost sales costs are considered to fit the realistic conditions of perishable or obsolete products.

Indeed, in general, the lost sales incur a cost corresponding to the lost revenue (selling price) and another related to the loss of customer goodwill at a later time. In our work, we assume that the lost sales cost is equal to the selling price, as the case of most research papers because estimating the future 
impact on customers is typically hard. The reader can refer to Chen and Zhang (2017) to see that the lost demand due to the cost of customer goodwill has not received much attention in the academic literature.

It is well known that the obsolete product loses its value through time because of rapid changes of technology, and this usually results in a decrease in their selling price. For example, the mobile phone loses a part of its primary selling price when there is an introduction of a new product. For a perishable product, the retailer can offer selling price discount over time (Goyal and Giri, 2001). All those observations allow us to consider NI lost sales costs in our assumptions.

We model LSP-BB with lost sales, FTL cost structure, and $w>1$ (LSP$\mathrm{BB}_{L S, F T L}$ with $w>1$ ) as a mixed integer linear program (MILP) taking into account all the features of the problem. The latter is the most general case of our problem. The number of $w$-time intervals in the planning horizon $1 . . T$ is denoted by $N$, with $N=\left\lfloor\frac{T}{w}\right\rfloor$ and $1 \leq N \leq \frac{T}{2}$. The problem with the fractional $\frac{T}{w}$ is solved as the one with the integer $\frac{T}{w}$. The only difference with the fractional $\frac{T}{w}$ is the last interval being smaller than the others. We consider that at the end of each period $i w$ with $1 \leq i \leq N$, the retailer returns the quantity $q_{i w}$ to the supplier. The total cost of the related problem depends on the decision of when and how much quantities to order, to return to the supplier, to lose and to store. The MILP for the $\operatorname{LSP}_{-B S, F T L}$ with $w>1$, defined by (1)-(10) and denoted as $P_{B B}$ is given below, whose objective is to minimize the total $\operatorname{cost} \mathscr{C}$ of setup, procurement, inventory and shortage taking into account return revenues. 


$$
\mathrm{P}_{B B}\left\{\begin{array}{lll}
\min \mathscr{C}=\sum_{t=1}^{T}\left(f_{t} y_{t}+\left(p_{t} x_{t}+a_{t} A_{t}\right)+h_{t} s_{t}+l_{t} L_{t}-p_{t}^{b} q_{t}\right) \\
\text { s.t. } \quad x_{t}+s_{t-1}=d_{t}-L_{t}+s_{t}+q_{t} & \forall t=1, \ldots, T \\
& q_{t}=0 & \\
& & t \neq i w \text { with } \\
& & i=1, \ldots, N \\
& s_{i w}=0 & \\
s_{0}=0 & \forall i=1, \ldots, N \\
L_{t} \leq d_{t} & \forall t=1, \ldots, T \\
& x_{t} \leq\left\lceil\frac{\sum_{j=t}^{T} d_{j}}{V_{t}}\right\rceil V_{t} y_{t} & \forall t=1, \ldots, T \\
& x_{t} \leq A_{t} V_{t} & \forall t=1, \ldots, T \\
& x_{t}, s_{t}, q_{t}, L_{t} \in \mathbb{R}_{+} & \forall t=1, \ldots, T \\
& A_{t} \in \mathbb{N}, y_{t} \in\{0,1\} & \forall t=1, \ldots, T
\end{array}\right.
$$

Constraints (2) are the balancing constraints linking inventory, ordering quantity, returned quantity, demand and lost sales quantities for all periods. In Constraints (3), no returns of unsold goods are permitted for the periods different from $i w$. In every $w$ periods, we do not talk about stored goods but rather about returned goods, that's why Constraints (4) ensure that the stock levels are equal to zero for those periods. Therefore, our problem is decomposed into $N$ independent problems with these constraints. Constraint (5) provides the initial inventory value as zero owing to problem simplification. Constraints (6) make sure that any amount of unmet demand $L_{t}$ in period $t$ cannot exceed the demand $d_{t}$ of that period. Constraints (7) ensure the setup variable generation in which the procurement variable cannot exceed the maximum quantity purchased from that period till $T$. Constraints (8) are for the batch replenishment which means that there are sufficient pallets containing the product quantity in each period. The remaining constraints (9) and (10) are nonnegativity, integrality and binary constraints on the decision variables. 


\section{LSP-BB without lost sales}

The unused items are returned to the supplier, without lost sales, at the end of each period $i w$ with $i=1, \ldots, N, N=\frac{T}{w}, N \in \mathbb{N}$ and $1 \leq w \leq T$. If $\frac{T}{w} \in \mathbb{R}_{+}$, then this problem is solved in the same way as the one with $\frac{T}{w} \in \mathbb{N}$. Therefore, there are $N$ independent problems to solve related to each subproblem $\llbracket(i-1) w+1, i w \rrbracket \forall i=1, \ldots, N$ (see Figure 4$)$. We optimize each subproblem as $\llbracket 1, w \rrbracket$. The total cost of the initial problem is computed as the sum of these $N$ independent problems's costs.

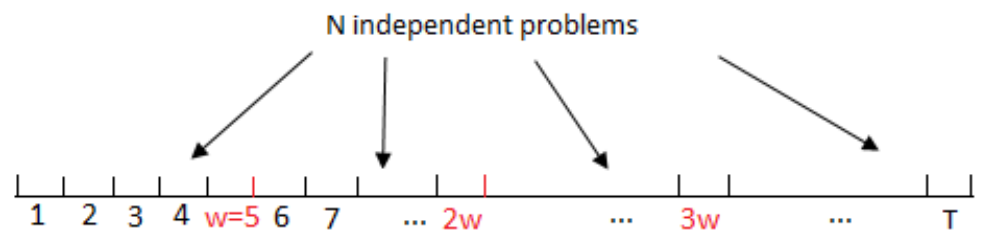

Figure 4: The $N$ intervals of our problem (illustration for $w=5$ )

For the return policy $w=1$, the left-over items are sold to the supplier at the end of each period $t$, thus, there is no remaining stock at retailer level over the whole horizon $\left(s_{t}=0, t=1, \ldots, T\right)$. Note that without any stock linking the periods, we can no more call this problem as "lot sizing". Indeed, there are $T$ independent problems to solve, one separate problem for each period $t, t=1, \ldots, T$ to optimize. Thus, in the following, we denote this latter as single period problem, SP-BB, instead of LSP-BB with $w=1$. Furthermore, we consider in this return policy that we have a full return with time-dependent batch sizes, and a unit return revenue lower than a unit procurement cost in every period. 
For the return policy $w>1$, we have $N$ independent problems to solve with $1 \leq N \leq \frac{T}{2}$. Besides, we restrict ourselves to the case with stationary batch size in order to propose exact polynomial time algorithms. Note that Akbalik and Rapine (2013) show that the uncapacitated batch lot sizing problem with time-dependent batch sizes is NP-hard if any one of the cost parameters (setup, fixed cost per batch, unit procurement cost or unit inventory holding cost) is allowed to be time-dependent. Thus, our problem is also NP-hard for the case with time-dependent batch sizes. Besides, in practical cases, the batch sizes do not really vary from one period to another. In the subproblem $\llbracket 1, w \rrbracket$, we also consider that the return revenue $p_{w}^{b}$ in period $w$ is strictly less than all the procurement costs $p_{t} \forall t \in\{1, \ldots, w\}$ to protect the supplier. We also consider the full return.

We study the four sub-cases, namely: SP-BB with only full batches, LSPBB with only full batches and $w>1$, SP-BB with FTL cost structure and finally LSP-BB with FTL cost structure and $w>1$.

\section{1. $S P-B B$ with only full batches (SP-BB $\left.B_{O F B}\right)$}

In $P_{B B}$, we set $w=1, l_{t}=+\infty, \forall t=1, \ldots, T$. Thus, we have to return unused products at the end of each ordering period. Besides, we alter Constraints (3) by $q_{t} \geq 0, \forall t=1, \ldots, T$, Constraints (4) by $s_{t}=0, \forall t=1, \ldots, T$ and Constraints (8) by $x_{t}=A_{t} V_{t}, \forall t=1, \ldots, T$. Consequently, we obtain the $\mathrm{SP}-\mathrm{BB}_{O F B}$. We state an important dominance property of this problem.

Property 1. An optimal plan $\pi^{*}$ orders the positive quantity $x_{t}^{*}=\left\lceil\frac{d_{t}}{V_{t}}\right\rceil V_{t}$ in every period $t \in\{1, \ldots, T\}$.

The proof of Property 1 can be found in Supplementary document. 
In the $\mathrm{SP}-\mathrm{BB}_{O F B}$, the retailer returns the surplus $q_{t}=\left\lceil\frac{d_{t}}{V_{t}}\right\rceil V_{t}-d_{t}$ to the supplier at the end of $t$. It is then easy to compute the total ordering cost without any optimization process as follows:

$$
\mathscr{C}^{*}{ }_{B B, O F B, w=1}=\sum_{t=1}^{T}\left(f_{t}\left\lceil\frac{d_{t}}{d_{t}+1}\right\rceil+p_{t}\left\lceil\frac{d_{t}}{V_{t}}\right\rceil V_{t}+a_{t}\left\lceil\frac{d_{t}}{V_{t}}\right\rceil-p_{t}^{b}\left(\left\lceil\frac{d_{t}}{V_{t}}\right\rceil V_{t}-d_{t}\right)\right)
$$

This cost is constant since it depends only on known cost parameters and $d_{t}, V_{t}$ values. It is easy to see that a linear time algorithm in $O(T)$ solves this planning problem to the optimality. This consists in determining $x_{t}^{*}$ by scanning each $t$ in $\{1, \ldots, T\}$ to have $x_{t}^{*}=\left\lceil\frac{d_{t}}{V_{t}}\right\rceil V_{t}$.

\section{2. $L S P-B B$ with only full batches and $w>1\left(L S P-B B_{O F B}\right.$ with $\left.w>1\right)$}

In $P_{B B}$, we assume $l_{t}=\infty, V_{t}=V, \forall t=1, \ldots, T$, and we modify Constraints (8) by $x_{t}=A_{t} V, \forall t=1, \ldots, T$ to obtain the LSP-BB $O F B$ with $w>1$. Recall that the $N$ independent problems are separately solved in order to compute the total optimal cost of $\operatorname{LSP}_{-B} B_{O F B}$ with $w>1$ by summing up individual costs of subproblems $\llbracket(i-1) w+1, i w \rrbracket, i=1, \ldots, N$. In what follows, we only consider how to solve the subproblem $\llbracket 1, w \rrbracket$ which can be applied to all subproblems. Li et al. (2004) study the LSP with batch ordering, time varying cost parameters and backlogging where the production quantity in each period is limited to a multiple of constant batch size $V$. The authors propose an algorithm that runs in $\mathrm{O}\left(T^{2}\right)$ time which is then lowered to $O(T \log (T))$ time through Monge matrices. In $\mathrm{LSP}_{-\mathrm{BB}_{O F B}}$ with $w>1$, the backlog is not allowed and the buyback contract is considered. Notice that we use a solution algorithm very close to that proposed by Li et al. (2004) 
with additional return quantities.

The idea of the algorithm is to detect the replenishment periods for determining the optimal total cost of $\llbracket 1, w \rrbracket$. Between two replenishment periods $i$ and $j$, we have to satisfy the demands without backlogging nor lost sales taking into consideration the stock levels at the beginning of period $i$ and at the end of period $j$. We define the following notations, similar to Li et al. (2004) which will be used in our approach:

$D_{i, j}$ : Cumulative demand from period $i$ to period $j$ if $i \leq j$.

$$
D_{i, j}= \begin{cases}\sum_{k=i}^{j} d_{k} & i \leq j \quad \forall i, \forall j \in\{1, \ldots, w\} \\ 0 & i>j\end{cases}
$$

$R_{j}$ : Minimum ending inventory level of period $j$ if we order in period 1 the minimal number of batches $\left\lceil\frac{D_{1, j}}{V}\right\rceil$ to cover the demand from period 1 to period $j$. For every $j$, we have $0 \leq R_{j} \leq V$.

$$
\begin{aligned}
& R_{0}=0 \\
& R_{j}=\left\lceil\frac{D_{1, j}}{V}\right\rceil V-D_{1, j} \quad \forall j \in\{1, \ldots, w\}
\end{aligned}
$$

For $j=1,2, \ldots, w-1$, the ending inventory level of period $j$ has the following form: $s_{j}=R_{j}+\alpha V$ with $\alpha \in \mathbb{N}$.

We now cite two suitable dominance properties to this problem.

Property 2. There exists an optimal solution such that, for any replenishment period $j$, the ending inventory value of period $j-1$ is equal to $R_{j-1}$ 
with $s_{j-1}=R_{j-1}, \forall j=1, \ldots, T$.

Property 2. is a special case of Property 2 stated in Li et al. (2004).

Property 3. In any optimal policy, the returned quantity of unused products at the end of period $w$ to the supplier is $q_{w}=R_{w}$.

The proofs of Properties 2 and 3 are in Supplementary document.

We will propose an algorithm which solves the $\operatorname{LSP}_{-B_{O F B}}$ with $w>1$ by using Properties 2 and 3 . The idea is to compute the optimal cost $C_{j}$ satisfying the demand in periods $1,2, \ldots, j$, given that period $j+1$ is a replenishment period with $1 \leq j \leq w-1\left(s_{j}=R_{j}\right)$, and $j$ is a return period with $j=w\left(s_{w}=0\right.$ and $\left.q_{w}=R_{w}\right)$. An $O\left(w^{2}\right)$ time dynamic programming algorithm is presented as follows in order to compute the optimal total cost

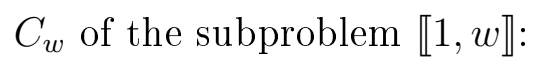

Recurrence relation. For $j=1,2, \ldots, w$,

$$
C_{j}=\min _{0 \leq i<j}\left\{C_{i}+f_{i+1}+\left(p_{i+1}+\frac{a_{i+1}}{V}\right)\left(R_{j}+D_{i+1, j}-R_{i}\right)+H_{i+1, j+1}\right\}
$$

Boundary condition. $C_{0}=0$

Objective. $C_{w}$

In this relation, the periods $i+1$ and $j+1$ are consecutive ordering periods. The number of batches acquired between periods $i+1$ and $j$ can be easily computed because we know the ending inventory level of period $i$ which is equal to $R_{i}$, and the one of period $j$ which is maintained at $R_{j}$. We add to the ordering cost at period $i+1$, the total holding cost $H_{i+1, j+1}$ from the end 
of period $i+1$ up to the beginning of period $j+1$ knowing that there is no replenishment between period $i+2$ and period $j$, and for $0 \leq i<j \leq w-1$ we have $s_{j}=R_{j}$ and $s_{w}=0$, and for $j=w$ we have $q_{w}=R_{w}$. All $H_{i+1, j+1}$ values can be obtained recursively in $O\left(w^{2}\right)$ time as follows with $\left|H_{w+1, w+1}\right|$ representing the return revenues in period $w$ :

$$
\begin{cases}H_{i+1, j+1}=H_{i+2, j+1}+h_{i+1}\left(D_{i+2, j}+R_{j}\right) & \text { if } 0 \leq i<j \leq w \\ H_{j+1, j+1}=0 & \text { if } 1 \leq j \leq w-1 \\ H_{w+1, w+1}=-p_{w}^{b} R_{w} & \text { if } j=w\end{cases}
$$

Since there are $\frac{T}{w}$ independent problems, the complexity of the whole LSP$\mathrm{BB}_{O F B}$ with $w>1$ is computed in $O(T w)$ time with $2 \leq w \leq T$ which is less than the $O\left(T^{2}\right)$ time algorithm of Li et al. (2004) because of the integration of buyback contract into LSP.

\section{3. $S P-B B$ with FTL cost structure $\left(S P-B B_{F T L}\right)$}

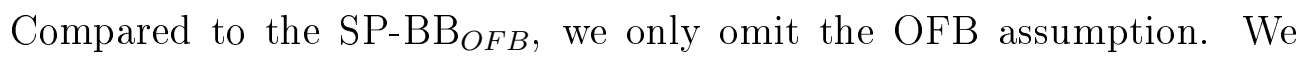
present an important optimality property of this problem.

Property 4. The optimal ordered quantity $x_{t}^{*}$ is exactly the demand $d_{t}$ in every period $t$.

The proof of Property 4 can be found in Supplementary document.

In the SP-BB $F T L$, there is, thus, never a surplus at the end of return periods, which means that $q_{t}=0, \forall t=1, \ldots, T$. The total ordering cost of the $\mathrm{SP}-\mathrm{BB}_{F T L}\left(\mathscr{C}^{*}{ }_{B B, F T L, w=1}\right)$ is a constant, which is given as follows: 


$$
\mathscr{C}^{*}{ }_{B B, F T L, w=1}=\sum_{t=1}^{T}\left(f_{t}\left\lceil\frac{d_{t}}{d_{t}+1}\right\rceil+p_{t} d_{t}+a_{t}\left\lceil\frac{d_{t}}{V_{t}}\right\rceil\right)
$$

One can obtain the optimal plan using a linear time algorithm by assigning to $x_{t}^{*}$ for each $t$ in $\{1, \ldots, T\}$ the demand $d_{t}$.

\subsection{LSP-BB with FTL cost structure and $w>1$ ( $L S P-B B_{F T L}$ with $\left.w>1\right)$}

The LSP-BB $B_{F T L}$ with $w>1$ is the general model of $P_{B B}$ with constant batch size and without lost sales. We again solve each subproblem $\llbracket(i-1) w+1, i w \rrbracket$, for all $i \in\{1, \ldots, N\}$ as previously. We assume a linear full-truckload cost function with linear variable procurement costs. $\mathrm{Li}$ et al. (2004) develop an $\mathrm{O}\left(T^{3} \log T\right)$ time algorithm for the dynamic lot sizing with fractional batch ordering (LSP-B) and backlogging. The authors assume non-decreasing concave holding, backlogging, and Less-than-Truck Load (LTL) freight cost functions and linear variable procurement costs. Akbalik and Rapine (2018) study a similar LSP-B but without backlogging. They suppose linear holding costs, but a LTL cost function with concave variable costs. Thereafter, the authors propose an $\mathrm{O}\left(T^{4}\right)$ time algorithm for LSP-B without backlogging but with concave procurement costs being more general than in Li et al. (2004).

We decided to adapt the idea of the Akbalik and Rapines' (2018) algorithm to our subproblem $\llbracket 1, w \rrbracket$ with buyback contract, because it is much simpler to implement and to understand. It will also be used in section 5.4 as one of the solutions of our problem with lost sales and batch ordering. As defined in the literature, a period $i$ is a regeneration period if it has a null entering stock level $\left(s_{i-1}=0\right)$. Furthermore, $(u, v)$ is called a subplan if 
periods $u$ and $v$ are consecutive regeneration periods such that $u<v$ and for each period $t=u, \ldots, v-2$, we have $s_{t}>0$. The idea of the algorithm is to compute the optimal cost $\mathscr{C}(u, v)$ of each possible subplan $(u, v)$ to satisfy the demand in periods $u, u+1, \ldots, v-1$, such that $1 \leq u<v \leq w+1$ and finally to compute the total optimal cost using a Shortest Path (SP) algorithm. Define $C_{v-1}$ as the optimal total cost to satisfy the demand in periods $1,2, \ldots, v-1$ given that period $v$ is a regeneration period $(1 \leq v \leq w+1)$. The following SP algorithm computes the total cost $C_{w}$ :

For $v=2,3, \ldots, w+1, \quad C_{v-1}=\min _{1 \leq u<v}\left\{C_{u-1}+\mathscr{C}(u, v)\right\} \quad$ such that $C_{0}=0$

The running time of the above $\mathrm{SP}$ algorithm is $O\left(w^{2}\right)$ if all the $\mathscr{C}(u, v)$ values have been predetermined. Akbalik and Rapine (2018) show that finding the minimal cost value of a subplan $(u, v)$ is performed in time complexity $O\left(w^{2}\right)$. This implies that the subproblem $\llbracket 1, w \rrbracket$ is solvable in $O\left(w^{4}\right)$ time and LSP-BB $F T L$ with $w>1$ in $O\left(T w^{3}\right)$ time.

In each subplan $(u, v), t$ and $r$ are two ordering periods such that $u \leq$ $t<r \leq v$. If there is at most one ordering period between $t$ and $r$, then the set $\{t, t+1, \ldots, r-1\}$ is called a replenishment cycle. We denote by $[t, r]^{u, v}$ such a cycle. The calculation of $\mathscr{C}(u, v)$ requires to decompose the subplan $(u, v)$ into different forms of replenishment cycles $[t, r]^{u, v}$. Akbalik and Rapine (2018) (pages 9-11, Appendix A) describe how the optimal cost $\mathscr{L}[t, r]^{u, v}$ of each replenishment cycle $[t, r]^{u, v}$ such that $t$ and $r$ are full batch ordering periods can be computed in constant time. In our paper, we explain how to compute the following costs by presenting a numerical example in Section 4.4.4:

- The optimal cost $\mathscr{L}[u, t]^{u, v}$ such that $u$ is a fractional batch ordering 
period with $0<x_{u}<V, t$ is a full batch ordering period and $u$ and $t$ are consecutive replenishment periods which means that no other ordering period exists between $u$ and $t$,

- The optimal cost $\mathscr{L}[t, r]^{u, v}$ such that $t$ is a fractional batch ordering period with $x_{t} \geq V, r$ is a full batch ordering period and $t$ and $r$ are consecutive replenishment periods,

- The optimal cost $\mathscr{C}(u, v)$.

\subsubsection{Definitions and properties}

For the LSP-BB ${ }_{F T L}$ with $w>1$, before defining the dominance properties related to batch ordering, we present the major property related to the buyback contract.

Property 5. The quantity returned to the supplier at the end of period $w$ is null $\left(q_{w}^{*}=0\right)$.

A proof for this property is stated in Supplementary document.

Property 6. (Lee et al., 2003) There is an optimal policy $\pi^{*}$, in which, there exists at most one fractional batch ordered in any of its subplans. It means that, for a subplan $(u, v)$, there is at most one fractional batch replenished among periods $u, u+1, \ldots, v-1$.

Note that Lee et al. (2003) are the pioneer having stated the same property for their model. Property 6 can be proven by using the proof of Property 4, in Li et al. (2004). In the following, we introduce the definition of a large ordering period which is proposed by Akbalik and Rapine (2018) that will be used in Property 7. 
Definition 1. (Akbalik and Rapine, 2018, page 9) An ordering period $t$ is called a large period if the retailer procures at least one full batch (i.e. $\left.x_{t} \geq V\right)$, and is called small $\left(x_{t}<V\right)$ otherwise.

By Property 6 and Definition 1, there is at most one small ordering period over the subplan $(u, v)$, which corresponds to the ordering of a fractional batch. Nevertheless, the period of fractional batch ordering can be also large if this fractional batch is ordered in addition to full batches. We have the following property which is used to solve the LSP-B first stated in Akbalik and Rapine (2018).

Property 7. (Akbalik and Rapine, 2018, page 10) Consider that the periods $t$ and $r$ are two consecutive ordering periods. In an optimal policy $\pi^{*}$, if both periods are large, then $s_{r-1}<V$. In other words, if $s_{r-1} \geq V$ then either the period $t$ is large and $r$ is small, or vice versa.

We present, in Supplementary document, a different way to prove Property 7 than the one proposed by Akbalik and Rapine (2018) knowing that we consider unit procurement costs.

Property 8. If there is a small ordering period $\bar{t}$ between two large consecutive ordering periods $t$ and $r(t<\bar{t}<r)$, then $s_{r-1}<2 V$.

Refer to Supplementary document in order to see how Property 8 is proved.

4.4.2. How to compute $\mathscr{L}[t, r]^{u, v}$ in the subplan $(u, v)$

In a given subplan $(u, v)$, we need to compute the cost $\mathscr{L}[t, r]^{u, v}$ to satisfy the demands $d_{t}, d_{t+1}, \ldots, d_{r-1}$ of the replenishment cycle $[t, r]^{u, v}$ in different situations. Recall that, there is at most one fractional batch occurring over 
the time horizon $u, \ldots, v-1$, so we denote by $\bar{x}_{u, v}$ the fractional quantity ordered in the incomplete batch such that $\bar{x}_{u, v}=D_{u, v-1}-\left\lfloor\frac{D_{u, v-1}}{V}\right\rfloor V$ and by $\bar{t}_{u, v}$ the fractional ordering period such that $u \leq \bar{t}_{u, v}<v$. The period $\bar{t}_{u, v}$ can be a large or a small ordering period. Thereafter, we distinguish 3 cases according to the criterion and the position of $\bar{t}_{u, v}$ in $(u, v)$. In the first case, the subplan $(u, v)$ has a small period $\bar{t}_{u, v}$ such that $\bar{t}_{u, v}>u$. In the second case, $\bar{t}_{u, v}$ remains small but we have $\bar{t}_{u, v}=u$. In the last case, $\bar{t}_{u, v}$ is large. In each case, there are different situations. Finally, we can have 8 possible values of the cost $\mathscr{L}[t, r]^{u, v(i)}$ with $i=1, \ldots, 8$ according to different cases (see Table 3).

Table 3: Costs $\mathscr{L}[t, r]^{u, v}$ in different situations

\begin{tabular}{|c|c|c|c|}
\hline $\begin{array}{l}\text { Cases } \\
(u, v)\end{array}$ & \multicolumn{2}{|l|}{ Different situations of $[t, r]^{u, v}$} & $\begin{array}{l}\text { Possible } \\
\text { costs } \\
{[t, r]^{u, v}}\end{array}$ \\
\hline \multirow{5}{*}{$\begin{array}{l}\bar{t}_{u, v} \text { is small } \\
\bar{t}_{u, v}>u\end{array}$} & \multirow{2}{*}{$\begin{array}{l}\bar{t}_{u, v}<t<r \text { with } t \text { and } r \text { are large and } \\
\text { consecutive ordering periods }\end{array}$} & $s_{t-1}^{u, v}<V$ & $\mathscr{L}[t, r]^{u, v(1)}$ \\
\hline & & $V \leq s_{t-1}^{u, v}<2 V$ & $\mathscr{L}[t, r]^{u, v(2)}$ \\
\hline & \multirow{2}{*}{$\begin{array}{l}t<\bar{t}_{u, v}<r \text { with } t \text { and } r \text { are large } \\
\text { consecutive ordering periods }\end{array}$} & $s_{r-1}^{u, v}<V$ & $\mathscr{L}[t, r]^{u, v(3)}$ \\
\hline & & $V \leq s_{r-1}^{u, v}<2 V$ & $\mathscr{L}[t, r]^{u, v(4)}$ \\
\hline & \multicolumn{2}{|c|}{$t<r<\bar{t}_{u, v}$ with $t$ and $r$ are large and consecutive ordering periods } & $\mathscr{L}[t, r]^{u, v(5)}$ \\
\hline \multirow{2}{*}{$\begin{array}{l}\bar{t}_{u, v} \text { is small } \\
\bar{t}_{u, v}=u\end{array}$} & \multicolumn{2}{|c|}{$u<t$ with 2 consecutive ordering periods $u$ and $t$, and $t$ is large } & $\bar{L}[u, t]^{u, v(6)}$ \\
\hline & \multicolumn{2}{|c|}{$t$ and $r$ are large and consecutive ordering periods with $u<t<r$} & $\mathscr{L}[t, r]^{u, v(7)}$ \\
\hline $\bar{t}_{u, v}$ is large & \multicolumn{2}{|c|}{$\bar{t} t=\bar{t}_{u, v}$ and, $t$ and $r$ are large and consecutive ordering periods with $t<r$. } & $\mathscr{L}[t, r]^{u, v(8)}$ \\
\hline
\end{tabular}

All the ending inventory levels must be calculated for the periods $u, u+$ $1, \ldots, v-2$ because we have $s_{u-1}^{u, v}=s_{v-1}^{u, v}=0$. The calculation details of all the values of $\mathscr{L}[t, r]^{u, v(i)}$, for $1 \leq u \leq t<r \leq v \leq w+1$ and $i=1, \ldots, 8$, are presented in Supplementary document, and, these values can all be computed in $O\left(w^{4}\right)$ time. 


\subsubsection{How to compute $\mathscr{C}(u, v)$}

We show the computation of $\mathscr{C}(u, v)$ with $1 \leq u<v \leq w+1$. After the calculation of all the possible costs $\mathscr{L}[t, r]^{u, v(i)}$ in the subplan $(u, v)$ with $i=1, \ldots, 8$, we can now compute 6 possible values of the cost of subplan $(u, v)$ according to different situations (see Table 4). The calculation details of $\mathscr{C}_{i}(u, v)$ for $i=1, \ldots, 6$ are given in Supplementary document.

Table 4: Costs $\mathscr{C}(u, v)$ in different situations

\begin{tabular}{|c|c|c|c|}
\hline \multicolumn{3}{|c|}{ Different situations of $(u, v)$} & $\begin{array}{l}\text { Possible } \\
\text { costs of }\end{array}$ \\
\hline \multirow{3}{*}{$\bar{t}_{u, v}$ is small } & \multirow{2}{*}{$\begin{array}{l}u \leq t<\bar{t}_{u, v}<r, t \text { and } r \text { are } 2 \\
\text { large consecutive ordering } \\
\text { periods }\end{array}$} & $\begin{array}{l}\bar{t}_{u, v} \text { avoids a shortage between } t \text { and } r \\
\text { with } r \leq v\end{array}$ & $\mathscr{C}_{1}(u, v)$ \\
\hline & & $\begin{array}{l}\bar{t}_{u, v} \text { ensures a part of demand for peri- } \\
\text { ods } r \text { and thereafter with } r<v\end{array}$ & $\mathscr{C}_{2}(u, v)$ \\
\hline & \multicolumn{2}{|l|}{$\bar{t}_{u, v}=u$} & $\mathscr{C}_{3}(u, v)$ \\
\hline \multirow{3}{*}{$\bar{t}_{u, v}$ is large } & \multicolumn{2}{|l|}{$\bar{t}_{u, v}=u$} & $\mathscr{C}_{4}(u, v)$ \\
\hline & \multirow{2}{*}{\multicolumn{2}{|c|}{$\begin{array}{l}\bar{t}_{u, v} \text { is the last ordering period in }(u, v) \\
u<\bar{t}_{u, v}<\text { last ordering period in }(u, v)\end{array}$}} & $\mathscr{C}_{5}(u, v)$ \\
\hline & & & $\mathscr{C}_{6}(u, v)$ \\
\hline
\end{tabular}

After the calculation of all possible values of $\mathscr{C}(u, v)$ which can be computed in $O\left(w^{4}\right)$, the latter is obtained as follows:

$$
\mathscr{C}(u, v)=\min _{1 \leq i \leq 6}\left\{\mathscr{C}_{i}(u, v)\right\}
$$

The aim is to compute the optimal cost $C_{w}$ using the SP algorithm as mentioned in Formula (11). Thus, it solves the subproblem $\llbracket 1, w \rrbracket$ in $O\left(w^{4}\right)$ time and the LSP-BB $F T L$ with $w>1$ in $O\left(T w^{3}\right)$.

\subsubsection{Numerical example for $L S P-B B_{F T L}$ with $w>1$}

We present an illustrative example of the algorithm of LSP-BB $\mathrm{BTTL}_{F}$ with $w>1$. We consider a time horizon of 4 periods in which the demands have 
to be satisfied in full and/or fractional batches of size $V=12$. Table 5 contains the data on the demand, cost and revenue. In the buyback contract considered in this example, we suppose that the unused items can be returned to the supplier only at the end of period 4. However, with Property 5 there is no return throughout the horizon.

Table 5: Demand, different costs and revenue data

\begin{tabular}{lllllll}
\hline $\begin{array}{l}\text { Period } \\
t\end{array}$ & $d_{t}$ & $f_{t}$ & $p_{t}$ & $a_{t}$ & $h_{t}$ & $p_{t}^{b}$ \\
\hline 1 & 75 & 100 & 13 & 23 & 1 & - \\
2 & 9 & 250 & 10 & 23 & 1 & - \\
3 & 50 & 0 & 21 & 4 & 1 & - \\
4 & 23 & 0 & 13 & 3 & 1 & 9 \\
\hline
\end{tabular}

Table 6 shows the optimal plan of our example indicating that there is one independent subproblem $\llbracket 1,4 \rrbracket$ to optimize. It is interesting to note that there are two subplans $(1,4)$ and $(4,5)$. In $(1,4)$, there are a large ordering period (full) placed in period 1 and a small ordering period (fractional) situated in period 3. Therefore, this subplan belongs to the Case 1 (especially the Sub-case 1.2) by considering that the period 4 is a dummy large ordering period. We have $s_{3}^{1,4}=0<V$, thus the optimal cost of the subplan $(1,4)$ is $\mathscr{L}[1,4]^{1,4(3)}$. Concerning the subplan $(4,5)$, there is one ordering period placed in period 4 which is large and fractional. Hence, we take into account the Case 3 by considering that the periods 4 and 5 are large and consecutive ordering periods. The optimal cost of the subplan $(4,5)$ is $\mathscr{L}[4,5]^{4,5(1)}$. 
Table 6: The optimal quantities of LSP-BB ${ }_{F T L}$ with $w>1$

\begin{tabular}{lllll}
\hline $\begin{array}{l}\text { Period } \\
t\end{array}$ & $x_{t}$ & $s_{t}$ & $q_{t}$ & $A_{t}$ \\
\hline 1 & 132 & 57 & 0 & 11 \\
2 & 0 & 48 & 0 & 0 \\
3 & 2 & 0 & 0 & 1 \\
4 & 23 & 0 & 0 & 2 \\
\hline
\end{tabular}

\section{LSP-BB with lost sales}

In LSP-BB with lost sales, demands can be partially or totally lost. More accurately, lost sales involve allowing some orders not to be delivered if the total cost of ordering this demand is prohibitive. We also study the four sub-cases listed in section 4 under the concept of lost sales: SP-BB with lost sales and only full batches; LSP-BB with lost sales, only full batches and $w>1$; SP-BB with lost sales and FTL cost structure; and finally LSP-BB with lost sales, FTL cost structure and $w>1$. For these four sub-cases, we consider the same assumptions mentioned in section 4 and we add the lost sales assumption wlog: $l_{t} V_{t} \geq p_{t} V_{t}+a_{t}, \forall \mathrm{t}=1, \ldots, T$. For the return policy $w>1$, we consider non-increasing lost sales costs over time. We recall that for $w=1$, we solve $T$ independent problems, each problem corresponding to one period. For $w>1$, there are $N$ independent problems to address. Each problem is expressed as $\llbracket(i-1) w+1, i w \rrbracket \forall i=1, \ldots, N$.

\section{1. $S P-B B$ with lost sales and only full batches ( $\left.S P-B B_{L S, O F B}\right)$}

The SP-BB ${ }_{L S, O F B}$ is a special case of $P_{B B}$. We fix $w$ to 1 and we modify Constraints (3) by $q_{t} \geq 0, \forall t=1, \ldots, T$, Constraints (4) by $s_{t}=0, \forall t=$ $1, \ldots, T$ and Constraints (8) by $x_{t}=A_{t} V_{t}, \forall t=1, \ldots, T$. We demonstrate 
two important dominance properties of this problem.

Property 9. There is an optimal solution such that a non-negative quantity is returned to the supplier in period $t \in\{1 . . T\}$ if and only if the lost sales amount drops to zero in that period. In our model, it is prohibited to have $\operatorname{both}\left(L_{t}^{*}=0\right.$ and $q_{t}^{*}=0$ if $\left.d_{t} \bmod V_{t} \neq 0\right)$ or both $\left(L_{t}^{*}>0\right.$ and $\left.q_{t}^{*}>0\right)$.

Property 10. The optimal policy $\pi^{*}$ orders a quantity $x_{t}^{*}$ which can take, in every period $t$, one of the three following values: $x_{t}^{*(1)}=0$, or $x_{t}^{*(2)}=\left\lfloor\frac{d_{t}}{V_{t}}\right\rfloor V_{t}$, or $x_{t}^{*(3)}=\left\lceil\frac{d_{t}}{V_{t}}\right\rceil V_{t}$.

For the proofs of properties 9 and 10, refer to Supplementary document.

Properties 9 and 10 permit us to develop an efficient algorithm in order to find the optimal plan for the $\mathrm{SP}-\mathrm{BB}_{L S, O F B}$. The key idea is to divide the time horizon into $T$ independent stages. Each stage corresponds to a period $t$, in which, by Property 10, the retailer procures either 0 or $\left\lfloor\frac{d_{t}}{V_{t}}\right\rfloor V_{t}$ or $\left\lceil\frac{d_{t}}{V_{t}}\right\rceil V_{t}$. Then, by Property 9 and following the constraints of our problem, we can compute $y_{t}^{*}, A_{t}^{*}, L_{t}^{*}$ and $q_{t}^{*}$ related to each possible optimal ordered quantity $x_{t}^{*}$ in every period $t$. For example, if $x_{t}^{*}=0$, then by using Constraints (2), we obtain $d_{t}-L_{t}^{*}+q_{t}^{*}=0$. Using Property 9 , if $L_{t}^{*}=0$ then $q_{t}^{*}=-d_{t}$ which contradicts the feasibility constraint of $q_{t}$, and if $q_{t}^{*}=0$ then $L_{t}^{*}=d_{t}$ which is trivial. After that, we calculate the total cost of each possible optimal ordered quantity $x_{t}^{*}$ in every period $t$. Hence, in each period $t$, we have three total costs $C_{t}^{(1)}, C_{t}^{(2)}$ and $C_{t}^{(3)}$ which correspond to the three possible optimal 
procurement quantities $x_{t}^{*(1)}, x_{t}^{*(2)}$ and $x_{t}^{*(3)}$ respectively.

$$
\begin{aligned}
C_{t}^{(1)} & :=l_{t} d_{t} \\
C_{t}^{(2)} & :=f_{t}+p_{t}\left\lfloor\frac{d_{t}}{V_{t}}\right\rfloor V_{t}+a_{t}\left\lfloor\frac{d_{t}}{V_{t}}\right\rfloor+l_{t}\left(d_{t}-\left\lfloor\frac{d_{t}}{V_{t}}\right\rfloor V_{t}\right) \\
C_{t}^{(3)} & :=f_{t}+p_{t}\left\lceil\frac{d_{t}}{V_{t}}\right\rceil V_{t}+a_{t}\left\lceil\frac{d_{t}}{V_{t}}\right\rceil-p_{t}^{b}\left(\left\lceil\frac{d_{t}}{V_{t}}\right\rceil V_{t}-d_{t}\right)
\end{aligned}
$$

Besides, we must choose the minimum cost. We define $C^{\text {min }}(t)$ as the minimum cost among $C_{t}^{(1)}, C_{t}^{(2)}$ and $C_{t}^{(3)}$ in every period $t$.

$$
\begin{aligned}
& C^{\min }(t):=\min \left\{C_{t}^{(1)}, C_{t}^{(2)}, C_{t}^{(3)}\right\} \\
& \mathscr{C}^{*}=\sum_{t=1}^{T} C^{\min }(t)
\end{aligned}
$$

We present $\mathscr{C}^{*}$ as the sum of $C^{\min }(t)$ from period 1 to period $T$. Consequently, $\mathscr{C}^{*}$ represents the optimal objective value of the $\mathrm{SP}-\mathrm{BB}_{L S, O F B} \cdot \mathscr{C}^{*}$ is computed by an $O(T)$ time algorithm.

5.2. $L S P-B B$ with lost sales, only full batches and $w>1$ (LSP-BB $B_{L S, O F B}$ with $w>1$ )

To obtain LSP-BB ${ }_{L S, O F B}$ with $w>1$, we substitute in $P_{B B}$ Constraints (8) by $x_{t}=A_{t} V$, with $V_{t}=V, \forall t=1, \ldots, T$. In this problem, we take into account the aspect of lost sales and we assume non-increasing lost sales costs over time. We note that the single-item LSP with immediate lost sales is studied by Aksen et al. (2003) which constitutes a special case of our problem with $w=T$ and $V_{t}=1$. They provide a dynamic programming algorithm with a complexity in $\mathrm{O}\left(T^{2}\right)$ time.

In our algorithm for $\operatorname{LSP}_{-B S} B_{L F B}$ with $w>1$, we use the same decomposition scheme into subplans $(u, v)$ as previously. Therefore, we must 
determine the minimum $\operatorname{costs} \mathscr{C}(u, v)$ of all subplans $(u, v)$ to decide how much of demand to satisfy and to lose for periods $u, u+1, \ldots, v-1$, with $1 \leq u<v \leq w+1$. Then, we can obtain the total optimal cost $C_{w}$ of the subproblem $\llbracket 1, w \rrbracket$, in which each demand can be either totally satisfied, or partially lost, or totally lost with $w+1$ being a regeneration period, by using the same SP algorithm proposed for $\mathrm{LSP}_{-\mathrm{BB}_{F T L}}$ with $w>1$ in section 4.4. In the following, we will prove that all the $\mathscr{C}(u, v)$ values can be obtained recursively in $O\left(w^{4}\right)$ for the subproblem $\llbracket 1, w \rrbracket$. Therefore, the overall complexity of the $\mathrm{LSP}_{-\mathrm{BB}_{L S, O F B}}$ with $w>1$ algorithm is $O\left(T w^{3}\right)$ time. In section 5.2.3, we present an illustrative example for this algorithm.

The key idea behind computing the $\operatorname{cost} \mathscr{C}(u, v)$ is to decompose the subplan $(u, v)$ into different cycles. At the beginning, we can find the replenishment cycles of type $[t, r]^{u, v(5)}$ such that $t$ and $r$ are 2 consecutive ordering periods without lost sales for $u \leq t<r \leq v$ (see Sub-case 1.3 in Supplementary document). After that, if $v<w+1$, then there is what we call a loss and replenishment cycle denoted by $\langle k, v\rangle^{u, v}$ such that $k$ is the last ordering period in $(u, v)$ and $v-1$ is the loss period in which the amount of unmet demand is strictly greater than 0 , for $u \leq k<v<w+1$. If $v=w+1$, then there is either one loss and replenishment cycle $\langle k, w+1\rangle^{u, w+1}$ for $u \leq k<w+1$, or one return and replenishment cycle denoted by $\|k, w+1\|^{u, w+1}$ such that $k$ is the last ordering period in $(u, w+1)$ and $w$ is the return period for $u \leq k<w+1$.

\subsubsection{Properties and corollaries}

We will first establish some optimality properties for our subproblem $\llbracket 1, w \rrbracket$ in order to propose an $O\left(w^{4}\right)$ time algorithm. 
We use Property 9 which affirms that $L_{w} q_{w}=0$. In other words, the retailer cannot return and lose at the same time. We give some other properties in the following:

Property 11. In any optimal solution, the starting inventory level $s_{r-1}$ of an ordering period $r$ is lower than $V$ units $\left(s_{r-1}<V\right)$, for $r=1, \ldots, w+1$.

Property 12. In any optimal solution, for a null ordering period $i\left(x_{i}=0\right)$, if $s_{i-1} \geq V$ then $L_{i}<V$.

Property 13. There exists an optimal policy such that $L_{t} s_{t}=0$ for each $t=1,2, \ldots, w$. If we decide not to satisfy a positive amount of demand in period $t$, the stock level will drop to zero at the end of that period. This also means that if we have a positive stock at the end of a period, the latter cannot be a lost sales period.

This property implies that in each subplan $(u, v)$, there is at most one loss period which is $v-1$, because this is the only period with a null outgoing stock level. Any possible lost sales quantity in the subplan $(u, v)$ must be of the form: $L_{v-1}^{u, v}=D_{u, v-1}-\left\lfloor\frac{D_{u, v-1}}{V}\right\rfloor V+n V$ such that $n \in \mathbb{N}$ and $n \leq\left\lfloor\frac{D_{u, v-1}}{V}\right\rfloor$. The proofs of Properties 11, 12 and 13 are stated in Supplementary document.

We have the following corollary which will be used to compute the total optimal cost of the subplan $(u, v)$ :

Corollary 1. If $v-1$ is an ordering period and there is a positive lost 
sales quantity, then $L_{v-1}^{u, v}=D_{u, v-1}-\left\lfloor\frac{D_{u, v-1}}{V}\right\rfloor V$ due to the lost sales assumption $l_{v-1} V \geq p_{v-1} V+a_{v-1}$ (Section 3). In addition, if $v-1$ is a non ordering period and $L_{v-1}>0$, then $L_{v-1}^{u, v}=d_{v-1}-s_{v-2}$. Corollary 1 is a result of all the $\mathrm{LSP}-\mathrm{BB}_{L S, O F B}$ with $w>1$ properties.

\subsubsection{How to compute $\mathscr{C}(u, v)$}

Recall that inside the subplan $(u, v)$ such that $1 \leq u<v \leq w+1$, there is at most one loss period which is the period $v-1$ and for any ordering period $t, s_{t-1}^{u, v}=\left\lceil\frac{D_{u, t-1}}{V}\right\rceil V-D_{u, t-1}$, based on the fact that there is no lost sales between the periods $u$ and $v-2$, and on Property 11. Furthermore, in each subplan $(u, w+1)$, the demand in period $w$ will be partially or fully lost if and only if the return is not made in that period, and if $D_{u, w} \bmod V \neq 0$. Otherwise, we have $L_{w}^{u, w+1} \geq 0$ if and only if $q_{w}=0$.

To find the optimal cost of the subplan $(u, v)$, we need to compute, first, the minimum total cost $G_{r}^{u, v}$ to satisfy the demands $d_{u}, d_{u+1}, \ldots, d_{r-1}$, for $1 \leq u<r \leq v \leq w+1$, such that $r$ must be the last ordering period and/or the loss or return period of $(u, v)$. The dynamic programming formulation of $G_{r}^{u, v}$ is given in Supplementary document.

In the case where $r$ is the last ordering period in the subplan $(u, v)$ and $v-1$ is the loss period with $L_{v-1}^{u, v}<V$, we have to compute, second, the total holding cost $H_{r}^{u, v}$ from the end of period $r$ until the beginning of $v-1$ to satisfy the demand of periods $r, r+1, \ldots, v-2$, for $u \leq r<v-1$. We define the formula of $H_{r}^{u, v}$ and the indicator functions $\mathbb{1}_{a}$ and $\mathbb{1}_{a_{i}}^{i}$ in Supplementary document.

After that, we can compute the possible values of $\mathscr{C}(u, v)$ in an $O\left(w^{4}\right)$ 
time. Thus, for $1 \leq u<v \leq w+1$ :

$$
\begin{aligned}
& \mathscr{C}(u, v)=\min \left\{\operatorname { m i n } _ { u \leq r \leq v - 1 \leq w } \left\{G_{r}^{u, v}+\min \left\{\mathbb{1}_{D_{r, v-1}-s_{r-1}^{u, v} \geq L_{v-1}^{u, v}} f_{r}+\left(p_{r}+\frac{a_{r}}{V}\right)\left\lfloor\frac{D_{r, v-1}-s_{r-1}^{u, v}}{V}\right\rfloor V\right.\right.\right. \\
& +H_{r}^{u, v}+l_{v-1} L_{v-1}^{u, v} \text {; } \\
& \left.\left.\mathbb{1}_{r=v-1} \mathbb{1}_{d_{v-1}-s_{v-2}^{u, v} \geq L_{v-1}^{u, v}} l_{v-1}\left|d_{v-1}-s_{v-2}^{u, v}\right|\right\}\right\} ; \\
& \left.\mathbb{1}_{v=w+1} G_{w+1}^{u, v}-p_{w}^{b}\left(\left\lceil\frac{D_{u, w}}{V}\right\rceil V-D_{u, w}\right)\right\}
\end{aligned}
$$

To see how the above relation is constructed, we consider in each subplan $(u, v)$ with $u<v$, that $u$ is the first ordering period and all the demands $d_{u}, d_{u+1}, \ldots, d_{r-1}$ must be satisfied with the minimum cost $G_{r}^{u, v}$ in which the period $r$ can be the last ordering period and/or the loss or return period, for $1 \leq u \leq r \leq v \leq w+1$. We have 3 situations of $(u, v)$. In the first situation, the subplan has a lost sales quantity less than the batch size $V$. Then, the retailer orders in period $r$ a quantity of $\left|\frac{D_{r, v-1}-s_{r-1}^{u, v}}{V}\right| V$ to satisfy the demands $d_{r}, d_{r+1}, \ldots, d_{v-2}$ and loses in $v-1$ a quantity of $L_{v-1}^{u, v}=D_{u, v-1}\left\lfloor\frac{D_{u, v-1}}{V}\right\rfloor V$. In this case, the position of the period $r$ belongs to $\{u, \ldots, v-1\}$ with $v-1 \leq w$. In the second situation, the lost sales quantity in $v-1$ is greater than or equal to $V$. Then, $v-1$ is a null ordering period and there is a loss of $d_{v-1}-s_{v-2}^{u, v}$. In this case, we have $r=v-1 \leq w$. In the third situation, the subplan $(u, v)$ possesses a procurement surplus and a return of $\left\lceil\frac{D_{u, w}}{V}\right\rceil V-D_{u, w}$. Then, we have $r=v=w+1$.

After predetermining all the possible $\mathscr{C}(u, v)$ values, we can compute the optimal total $\operatorname{cost} C_{w}$ to satisfy or to lose each demand in the subproblem $\llbracket 1, w \rrbracket$ with only full batch, expressed in Formula (11), in $O\left(w^{4}\right)$ time. Therefore, the running time of the dynamic programming algorithm proposed for $\mathrm{LSP}-\mathrm{BB}_{L S, O F B}$ with $w>1$ is $O\left(T w^{3}\right)$. 


\subsubsection{Numerical example for $L S P-B B_{L S, O F B}$ with $w>1$}

We use the same example presented for the algorithm of LSP-BB FTL $_{\text {with }}$ $w>1$ (see Section 4.4.4) by adding four lost sales costs, $l_{1}=23.5, l_{2}=22.3$, $l_{3}=21.8$ and $l_{4}=21.5$. In this problem, the demands can be partially or fully satisfied in full batches, or can be fully lost.

Table 7 shows the optimal solutions of this problem. We have the same decomposition of subplans $(1,4)$ and $(4,5)$. In $(1,4)$, the period 1 is the only ordering period which is large and full, and the period 3 is the only loss period in which the quantity of unmet demand is lower than the batch size 12. The optimal cost of the subplan $(1,4)$ is the cost of the loss and replenishment cycle $\langle 1,4\rangle^{1,4}$. In $(4,5)$, the period 4 is at the same time a large and full ordering period and a return period. The optimal cost of this subplan is the cost of the return and replenishment cycle $\|4,5\|^{4,5}$.

Table 7: The optimal quantities of LSP-BB ${ }_{L S, O F B}$ with $w>1$

\begin{tabular}{llllll}
\hline Period & $x_{t}$ & $s_{t}$ & $L_{t}$ & $q_{t}$ & $A_{t}$ \\
$t$ & & & & & \\
\hline 1 & 132 & 57 & 0 & 0 & 11 \\
2 & 0 & 48 & 0 & 0 & 0 \\
3 & 0 & 0 & 2 & 0 & 0 \\
4 & 24 & 0 & 0 & 1 & 2 \\
\hline
\end{tabular}

\subsection{SP-BB with lost sales and FTL cost structure ( $\left.S P-B B_{L S, F T L}\right)$}

Compared to the case $\mathrm{SP}-\mathrm{BB}_{L S, O F B}$, we do not consider any specific assumption on the batches. The ordered quantities can now be in fractional batches. Two important dominance properties are given as follows: 
Property 14. In any optimal solution, there is no returned quantity, i.e. $q_{t}^{*}=0, \forall t=1, \ldots, T$.

Property 15. The optimal plan $\pi^{*}$ orders either 0 or $\left\lfloor\frac{d_{t}}{V_{t}}\right\rfloor V_{t}$ or $d_{t}$ in every period $t$. We indicate the three possible optimal ordered quantities as follows: $x_{t}^{*(1)}=0, x_{t}^{*(2)}=\left\lfloor\frac{d_{t}}{V_{t}}\right\rfloor V_{t}$ and $x_{t}^{*(3)}=d_{t}$.

The proofs of Properties 14 and 15 are given in Supplementary document.

We develop an efficient algorithm for the SP-BB $B_{L S, F T L}$ that is based on Properties 14 and 15. The key idea of this problem is the same for the SP-BB $B_{L S, O F B}$. We have the following costs: $C_{t}^{(1)}, C_{t}^{(2)}, C_{t}^{(3)}, C^{\text {min }}(t)$ and $\mathscr{C}^{*}$ already defined in Section 5.1. We refer to Formulas (16)-(20) by just changing the quantity $x_{t}^{*(3)}$ from $\left\lceil\frac{d_{t}}{V_{t}}\right\rceil V_{t}$ to $d_{t}$ and subsequently the cost $C_{t}^{(3)}$ from $f_{t}+p_{t}\left\lceil\frac{d_{t}}{V_{t}}\right\rceil V_{t}+a_{t}\left\lceil\frac{d_{t}}{V_{t}}\right\rceil-p_{t}^{b}\left(\left\lceil\frac{d_{t}}{V_{t}}\right\rceil V_{t}-d_{t}\right)$ to $f_{t}+p_{t} d_{t}+a_{t}\left\lceil\frac{d_{t}}{V_{t}}\right\rceil$.

5.4. LSP-BB with lost sales, FTL cost structure and $w>1\left(L S P-B B_{L S, F T L}\right.$ with $w>1$ )

The LSP-BB ${ }_{L S, F T L}$ with $w>1$ is the most general problem with nonincreasing lost sales costs and a constant batch size. We will solve independently, in the same way as before, the subproblems $\llbracket 1, w \rrbracket, \llbracket w+1,2 w \rrbracket$, ..., $\llbracket T-w+1, T \rrbracket$ to obtain LSP-BB ${ }_{L S, F T L}$ with $w>1$. Our problem will be decomposed into subplans which start and end with zero stock. The optimal cost $C_{w}$ of the subproblem $\llbracket 1, w \rrbracket$ with ordering of full/fractional batches and lost sales can be computed in $O\left(w^{4}\right)$ by a shortest-path like dynamic programming algorithm used in section 4.4, given that all the possible subplan costs can be obtained in $O\left(w^{4}\right)$ time. Thus, we can compute the total optimal cost of LSP-BB ${ }_{L S, F T L}$ with $w>1$ with a complexity in $O\left(T w^{3}\right)$ time. The computation of the optimum cost of a subplan relies 
on the decomposition of $(u, v)$ into different types of cycles. We can find the different forms of replenishment cycles $[t, r]^{u, v(i)}$ for $i=1, \ldots, 8$ and $1 \leq u \leq t<r \leq v \leq w+1$ (see section 4.4) and/or the loss and replenishment cycle $\langle k, v\rangle^{u, v}$ for $1 \leq u \leq k<v \leq w+1$ (see section 5.2).

\subsubsection{Properties}

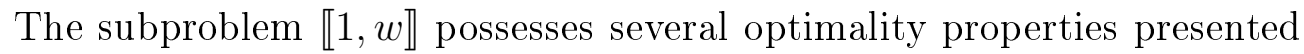
below. Properties 5, 6, 7, 8 (see section 4.4), and 13 (see section 5.2) remain valid for the general problem LSP-BB ${ }_{L S, F T L}$ with $w>1$.

Property 16. There exists an optimal solution, in which, for any subplan $(u, v)$ with $1 \leq u<v \leq w+1$, there is at most either one fractional batch ordered in one of its periods, or one loss period being the period $v-1$. This property is proved in Supplementary document.

Hence, if there is a positive lost sales quantity, then all ordering periods are large. Now, we can directly use Property 12 and Corollary 1.

\subsubsection{How to compute $\mathscr{C}(u, v)$}

All these properties imply that the cost of the subplan $(u, v)$ for $1 \leq u<$ $v \leq w+1$ in $\mathrm{LSP}_{-\mathrm{BB}_{L S, F T L}}$ with $w>1$ is the minimum between the cost $\mathscr{C}(u, v)$ in $\mathrm{LSP}_{-\mathrm{BB}} \mathrm{B}_{F L}$ with $w>1$, and the cost $\mathscr{C}(u, v)$ in $\mathrm{LSP}_{-\mathrm{BB}_{L S, O F B}}$ with $w>1$, but without taking into account the return revenue since the retailer can order fractional batches. Hence, the running time to compute all 
the values of $\mathscr{C}(u, v)$ is bounded by $O\left(w^{4}\right)$. They are obtained as follows:

$$
\begin{aligned}
& \mathscr{C}(u, v)=\min \left\{\min _{1 \leq i \leq 6}\left\{\mathscr{C}_{i}(u, v)\right\}\right. \\
& \min _{u \leq t \leq v-1 \leq w}\left\{G_{t}^{u, v}+\min \left\{\mathbb{1}_{D_{t, v-1}-s_{t-1}^{u, v} \geq L_{v-1}^{u, v}} f_{t}+\left(p_{t}+\frac{a_{t}}{V}\right)\left\lfloor\frac{D_{t, v-1}-s_{t-1}^{u, v}}{V}\right\rfloor V\right.\right. \\
& +H_{t}^{u, v}+l_{v-1} L_{v-1}^{u, v} \\
& \left.\left.\left.\mathbb{1}_{t=v-1} \mathbb{1}_{d_{v-1}-s_{v-2}^{u, v} \geq L_{v-1}^{u, v}} l_{v-1}\left|d_{v-1}-s_{v-2}^{u, v}\right|\right\}\right\}\right\}
\end{aligned}
$$

Once all these values are predetermined, the value of $C_{w}$ can be obtained in $O\left(w^{4}\right)$ time by using (11). Hence, the $\operatorname{LSP}_{-B_{L S, F T L}}$ with $w>1$ is solved in $O\left(T w^{3}\right)$ time.

\subsubsection{Numerical example for $L S P-B B_{L S, F T L}$ with $w>1$}

We consider the same example presented for the algorithm of LSP-BB ${ }_{L S, O F B}$ with $w>1$ (see Section 5.2.3) by ordering in full and/or fractional batches. The optimal decomposition of subplans is $(1,4)$ and $(4,5)$. The optimal cost of $(1,4)$ is the cost of the cycle $\langle 1,4\rangle^{1,4}$ as in the example of Section 5.2.3. In $(4,5)$, there is no return and the period 4 is a large and fractional ordering

period. Its optimal cost is the cost of the cycle $\mathscr{L}[4,5]^{4,5(1)}$ as in the example of Section 4.4.4.

\section{Conclusion and perspectives}

In this study, we considered the single-item lot sizing problem with batch ordering under the buyback contract. In our model, we merged the buyback contract into the LSP by considering that the retailer returns the unused units to the supplier either at the end of each period $t$, denoted by $w=1$ or at the end of every $w$ periods $w>1$ with full return and partial refund. Another option we studied is the possibility to not satisfy all the demands, 
knowing that losing a batch costs more than purchasing it. Therefore, for both LSP-BB without and with lost sales, we tackled 4 problems by assuming different hypotheses. In the first problem, we only consider full batches with $w=1$. The second problem is with $\mathrm{OFB}$ and $w>1$. To obtain the two problems that remain, we changed the OFB cost structure in the first two problems to the FTL cost structure.

In the LSP-BB without lost sales, we have developed 2 algorithms of complexity $O(T)$ for $w=1$ that solve the single period procurement planning with time-dependent size batch ordering according to the nature of cost structure (OFB or FTL). The key idea of these algorithms is to divide the problem with the planning horizon $1, \ldots, T$ into $T$ independent subproblems. When $w>1$, we have also developed 2 algorithms that solve LSP-BB with constant size batch ordering. The general idea of these algorithms is to divide the problems into $\frac{T}{w}$ subproblems. For the problem with OFB, the algorithm runs in $O(T w)$ time. The key idea of each subproblem which is close to the algorithm proposed by Li et al. (2004) is to detect the replenishment periods. For the problem with FTL costs, we have adapted the algorithm of Akbalik and Rapine (2018) to each subproblem to obtain an $O\left(T w^{3}\right)$ time algorithm. The idea is to precompute the fractional and full quantities replenished in each subplan.

For the LSP-BB with lost sales, we have developed 2 algorithms of complexity $O(T)$ for $w=1$, one for OFB and another for FTL cost structure. For the return policy $w>1$, we have proposed 2 algorithms that solve the LSP$\mathrm{BB}$ with constant size batch ordering and non-increasing lost sales costs. For the OFB cost structure, we have proposed an $O\left(T w^{3}\right)$ time algorithm. The 
key idea behind achieving this running time is to compute the full replenishment periods and lost sales quantities in each subplan. For the problem with FTL cost structure, we have developed an $O\left(T w^{3}\right)$ time algorithm. We compute the fractional, full and lost sales quantities in each subplan.

There are many interesting extensions of our topic to explore as perspectives. Such extensions might include another form of buyback contract in which the retailer can return products only in ordering periods with a maximum return percentage. For full return, we can try to propose an efficient algorithm, but for partial return, we can try to see if this problem is NPhard or not. Other interesting perspectives can be the multi-item and/or the multi-level cases. For instance, one can consider a system with multiple items arriving at a retailer in batches with the possibility of returns to the supplier in every period of the planning horizon. In each period, the total returned quantity of all the items may be limited by an amount specified by the supplier. For this problem, we can refer to two research axes: multi-item LSP with batch ordering and big-bucket LSP with several items sharing a common capacity for a given period. Given the increasing complexity of the related problems and the difficulty to propose efficient algorithms, one can try to develop a dynamic programming algorithm which is polynomial for a fixed number of items (Anily and Tzur, 2005), or use polyhedral approaches by proposing valid inequalities (see Akartunali et al., 2016; Doostmohammadi and Akartunali, 2018), relaxation or decomposition techniques (Fragkos et al. 2016), or heuristics (Absi et al. 2013) for a large dimension of items. Concerning the multi-level extension, one can imagine a system with serial suppliers replenishing an item to the upstream levels in some batches, with 
different levels of this chain having various buyback options. In this system, one can also consider intermediate demands occurring in each echelon (direct shipment to local customers), in addition to the demand of upstream levels. We can extend the work of Zhang et al. (2012) studying the multi-echelon LSP in series with intermediate demands, by adding the concepts of batch replenishment and/or return policy. One can try to see if the valid inequalities proposed by Zhang et al. (2012) can be adapted to our problem under some restrictive assumptions. Another interesting study on multi-level LSP is from van Vyve et al. (2014), which can be valuable to extend with our assumptions of buyback option and batch ordering. The system may be composed of one-warehouse facing dependent demand and multi-retailers facing independent demands, with inventories at both levels and retailers being able to return unused products in capacitated vehicles to the warehouse. One can see if the extended formulations proposed by the authors can be handled to integrate the new constraints specific to our problem.

\section{Acknowledgments}

We are grateful to Christophe Rapine for his careful reading and all his suggestions which helped to improve the presentation of this paper. We also thank to referees for their valuable comments and suggestions.

\section{References}

[1] Absi, N., S. Kedad-Sidhoum, S. Dauzère-Pérès, Uncapacitated lot-sizing problem with production time windows, early productions, backlogs and 
lost sales, International Journal of Production Research 49(9) (2011) $2551-2566$.

[2] Absi, N., B. Detienne, S. Dauzère-Pérès, Heuristics for the multi-item capacitated lot-sizing problem with lost sales, Computers $\&$ Operations Research 40(1) (2013) 264-272.

[3] Aggarwal, A., J.K. Park, Improved algorithms for economic lot-size problem. Operations Research 41(3) (1993) 549-571.

[4] Akartunali, k., I. Fragkos, A.J. Miller, T. Wu. Local cuts and two-period convex hull closures for big-bucket lot-sizing problems. INFORMS Journal on Computing 28(4) (2016) 766-780.

[5] Akbalik, A., Y. Pochet, Valid inequalities for the single-item capacitated lot sizing problem with stepwise costs, European Journal of Operational Research 198(2) (2009) 412-434.

[6] Akbalik, A., C. Rapine, Polynomial time algorithms for the constant capacitated single-item lot sizing problem with stepwise production cost, Operations Research Letters 40(5) (2012) 390-397.

[7] Akbalik, A., C. Rapine, The single item uncapacitated lot-sizing problem with time-dependent batch sizes: NP-hard and polynomial cases, European Journal of Operational Research 229(2) (2013) 353-363.

[8] Akbalik, A., A. B.Hadj-Alouane, N. Sauer, H. Ghribi, NP-hard and polynomial cases for the single-item lot sizing problem with batch ordering under capacity reservation contract, European Journal of Operational Research 257(2) (2017) 483-493. 
[9] Akbalik, A., C. Rapine, Lot sizing problem with multi-mode replenishment and batch delivery, Omega 81 (2018) 123-133.

[10] Aksen, D., K. Altinkemer, S. Chand, The single-item lot-sizing problem with immediate lost sales, European Journal of Operational Research, $147(3)(2003) 558-566$.

[11] Anily, S., M. Tzur, Shipping Multiple Items by Capacitated Vehicles: An Optimal Dynamic Programming Approach, Transportation Science $39(2)(2005)$ 233-248.

[12] Atamturk, A., D.S. Hochbaum, Capacity acquisition, subcontracting and lot-sizing, Management Science 47(8) (2001) 1081-1100.

[13] Bijvank, M., I.F.A. Vis, Lost-sales inventory theory: A review, European Journal of Operational Research 215 (2011) 1-13.

[14] Bitran, G.R., H.H. Yanasse, Computational complexity of the capacitated lot size problem, Management Science 28(10) (1982) 1174-1186.

[15] Bose I., P. Anand, On returns policies with exogenous price, European Journal of Operational Research 178 (2007) 782-788.

[16] Brahimi, N., N. Absi, S. Dauzère-Pérès, A. Nordli, Single-item dynamic lot-sizing problems: An updated survey, European Journal of Operational Research 263(3) (2017) 838-863.

[17] Buschkühl, L., F. Sahling, S. Helber, H. Tempelmeier, Dynamic capacitated lot-sizing problems: a classification and review of solution approaches, OR Spectrum 32(2) (2010) 231-261. 
[18] Chen, Z., and R. Zhang, Capital flow constrained lot sizing problem with loss of goodwill and loan, arXiv preprint arXiv:1708.08098 (2017).

[19] Dobos, I., A. Wimmer, Buyback and return policies for a book publishing firm, Working Paper, Vállalatgazdaságtan Intézet, Budapest, (2010).

[20] Doostmohammadi, M, K. Akartunali, Valid inequalities for two-period relaxations of big-bucket lot-sizing problems: Zero setup case, European Journal of Operational Research 267 (2018) 86-95.

[21] Federgruen, A., M.A. Tzur, A simple forward algorithm to solve general dynamic lot sizing models with $\mathrm{n}$ periods in $\mathrm{O}(\mathrm{nlogn})$ or $\mathrm{O}(\mathrm{n})$ time, Management Science 37(8) (1991) 909-925.

[22] Florian, M., J.K. Lenstra, A.H.G. Rinnooy Kan, Deterministic production planning: Algorithms and complexity, Management Science 26(7) (1980) 669-679.

[23] Fragkos, I, Z. Degraeve, B. De Reyck. A horizon decomposition approach for the capacitated lot sizing problem with setup times, INFORMS Journal on Computing, 28(3) (2016) 465-482.

[24] Goyal, S.K., B.C. Giri, Recent trends in modeling of deteriorating inventory, European Journal of Operational Research 134 (2001) 1-16.

[25] Hazra, J., B. Mahadevan, A procurement model using capacity reservation, European Journal of Operational Research 193(1) (2009) 303-316.

[26] He, Y., X. Zhao, Coordination in multi-echelon supply chain under sup- 
ply and demand uncertainty, International Journal of Production Economics 139 (2012) 106-115.

[27] Hou, J., A.Z. Zeng, L. Zhao, Coordination with a backup supplier through buy-back contract under supply disruption, Transportation Research Part E: Logistics and Transportation Review 46(6) (2010) 881895.

[28] Hwang, H.C., W. van den Heuvel, A.P.M. Wagelmans, The economic lotsizing problem with lost sales and bounded inventory, IIE Transactions 45(8) (2013) 912-924.

[29] Karimi, B., S.M.T. Fatemi Ghomi, J.M. Wilson, The capacitated lot sizing problem: a review of models and algorithms, Omega 31(5) (2003) $365-378$.

[30] Koca, E., H. Yaman, M.S. Akturk, Lot Sizing with Piecewise Concave Production Costs, INFORMS Journal on Computing 26(4) (2014) 767779 .

[31] Krishnan, H., R. Kapuscinski, D.A. Butz, Coordinating contracts for decentralized supply chains with retailer promotional effort, Management Science 50(1) (2004) 48-63.

[32] Lee, C.Y., S. Çetinkaya, W. Jaruphongsa, A dynamic model for inventory lot-sizing and outbound shipment scheduling at a third party warehouse, Operations Research 51(5) (2003) 735-747.

[33] Lee, C.Y., X. Li, Economic lot sizing: the capacity reservation model, Operations Research Letters 41(6) (2013) 670-674 
[34] Li, C.L., V.N. Hsu, W.Q. Xiao, Dynamic Lot Sizing with Batch Ordering and Truckload Discounts, Operations Research 52(4) (2004) 639-654.

[35] Padmanabhan, V., I.P.L Png, Returns Policies: Make Money by Making Good, Sloan Management Review/Fall (1995) 65-72.

[36] Padmanabhan, V., I.P.L Png, Manufacturer's Returns Policies and Retail Competition, Marketing Science 16(1) (1997) 81-94.

[37] Park, S.I., J.S. Kim, A mathematical model for a capacity reservation contract, Applied Mathematical Modelling 38(5-6) (2014) 1866-1880.

[38] Pasternack, B.A., Optimal pricing and returns policies for perishable commodities, Marketing Science 4(2) (1985) 166-176.

[39] Pochet, Y., L.A. Wolsey, Production planning by mixed integer programming, New York: Springer (2006).

[40] Sandbothe, R.A., G.L. Thompson, A forward algorithm for the capacitated lot size model with stockouts, Operations Research 38(3) (1990) $474-486$.

[41] Van Norden, L., S. Van de Velde, Multi-product lot-sizing with a transportation capacity reservation contract, European Journal of Operational Research 165(1) (2005) 127-138.

[42] Van Vyve, M., L. Wolsey, H. Yaman, Relaxations for two-level multiitem lot-sizing problems, Mathematical Programming 146(1-2) (2014) 495-523. 
[43] Wagner, H.M., T.M. Whitin, Dynamic version of the economic lot size model, Management Science 5(1) (1958) 89-96.

[44] Wagelmans, A., S. Van Hoesel, A. Kolen, Economic lot sizing: An $\mathrm{O}(\mathrm{n} \log (\mathrm{n}))$ algorithm that runs in linear time in the wagner whitin case, Operations Research 40(1) (1992) 145-156.

[45] Wu, D., Coordination of competing supply chains with news-vendor and buyback contract, International Journal of Production Economics 144(1) (2013) 1-13.

[46] Wu, S.D., M. Erkoc, S. Karabuk, Managing capacity in the high-tech industry: a review of literature, The Engineering Economist 50(2) (2005) $125-158$.

[47] Zhang, M., S. Küçükyavuz, H. Yaman. A polyhedral study of multiechelon lot sizing with intermediate demands. Operations Research, 60(4) (2012) 918-935. 